\title{
Lutte contre les ectoparasites des bovins par pédiluve : méthode innovante utilisée en zone périurbaine subhumide du Burkina Faso
}

\author{
F. Stachurski ${ }^{1 *}$ J. Bouyer ${ }^{2}$ F. Bouyer ${ }^{3}$
}

\begin{abstract}
Mots-clés
Bovin - Metastigmata - Glossinidae Méthode de lutte - Lutte intégrée Approche communautaire Innovation - Burkina Faso.
\end{abstract}

\begin{abstract}
Résumé
L'accroissement de la demande urbaine en protéines animales se traduit, entre autres, par le développement de filières bovines de production laitière dans les zones périurbaines d'Afrique de l'Ouest. Mais la maîtrise imparfaite des maladies et des parasites compromet la croissance de la production. Une technique de lutte innovante, basée sur le passage régulier dans un pédiluve contenant une formulation aqueuse d'un pyréthrinoïde, pourrait pourtant limiter l'impact des tiques et des glossines, principales contraintes pathologiques en zone subhumide. Cette méthode entraîne l'élimination de la plupart des adultes d'Amblyomma variegatum, la tique la plus nocive dans ces régions, avant leur fixation définitive. D'autre part, un tel traitement permet de diminuer fortement la population des glossines riveraines les plus représentées en Afrique occidentale (Glossina tachinoides et G. palpalis gambiensis) et donc de limiter I'incidence des trypanosomoses. Cette méthode assure ainsi une lutte rapide et efficace à coût réduit car la quantité de produit utilisé à chaque passage est faible. Mais la construction de l'installation, qui peut être utilisée par 400 à 600 animaux, représente un investissement non négligeable que des groupements d'éleveurs ou des investisseurs privés peuvent plus facilement prendre en charge que des éleveurs traditionnels isolés. La mise en place de cette méthode peut ainsi être envisagée dans le cadre d'une politique de développement et d'aménagement. L'intérêt et les modalités de l'appui technique nécessaire sont présentés. Au Burkina Faso, un projet d'appui au renforcement des organisations professionnelles d'éleveurs modernes (Ariope) a de la sorte financé quinze installations dans les zones périurbaines de Bobo-Dioulasso et Ouagadougou.
\end{abstract}

\section{INTRODUCTION}

L'accroissement de la demande urbaine en protéines animales se traduit entre autres par le développement de filières de production laitière dans les zones périurbaines d'Afrique de l'Ouest et centrale $(12,18)$. Dans ces périmètres, la majorité des élevages ayant

\footnotetext{
1. Cirad, UPR Contrôle des maladies, Montpellier, F-34000 France ; Cirdes, Bobo-Dioulasso, Burkina Faso.

2. Cirad, UPR Epidémiologie, Montpellier, F-34000 France ;

Cirdes, Bobo-Dioulasso, Burkina Faso.

3. Projet Appui au renforcement institutionnel des organisations professionnelles d'éleveurs modernes, ambassade de France, Ouagadougou, Burkina Faso.

* Auteur pour la correspondance

Cirad, département Emvt, TA30/D, Campus international de Baillarguet,

34398 Montpellier Cedex 5, France.

Tél. : +33 (0)4 67615800 , poste 4235 ; fax : +33 (0)4 67593798

E-mail : frederic.stachurski@cirad.fr
}

une vocation laitière sont de type sédentaire ou semi-sédentaire, et la charge animale des pâturages est fréquemment élevée (11). Ces caractéristiques, ainsi que la réduction de la densité de la faune sauvage dans ces zones fortement anthropisées (la lutte contre les glossines par traitement épicutané suppose que plus de 80 p. 100 des hôtes soient traités dans une zone donnée, ce qui n'est possible qu'en l'absence de faune sauvage), sont favorables à la mise en œuvre d'une nouvelle technique de lutte contre les tiques et les trypanosomoses qui constituent les principales contraintes pathologiques et limitent la production bovine, notamment laitière. Le regroupement des éleveurs en organisations professionnelles ainsi qu'un meilleur accès au crédit permettent en effet de prendre en considération des techniques nécessitant un investissement initial assez important mais assurant une diminution ultérieure des coûts de l'effort de lutte. Ces investissements peuvent également faire l'objet d'appuis de la part des gouvernements ou de projets de développement dans le cadre de l'aménagement périurbain et des politiques de développement. 
La maîtrise imparfaite de la pathologie bovine compromet la croissance de la production nécessaire pour accompagner l'augmentation de la population urbaine. En zone subhumide d'Afrique de l'Ouest, les contraintes pathologiques sont principalement représentées par les parasites et singulièrement par les glossines et les tiques, notamment l'espèce Amblyomma variegatum. Les mouches tsé-tsé transmettent les trypanosomes qui touchent 47 millions de bovins dans 37 pays et entraînent des pertes économiques inestimables (16). Les trypanosomoses restent des menaces présentes aux portes de nombreuses agglomérations urbaines et provoquent une diminution des performances zootechniques (production laitière et croissance altérées, mortalité et avortements accrus) et motrices (surface cultivée par un bœuf) pouvant entraîner indirectement une réduction de 2 à 10 p. 100 de la production agricole intérieure brute dans les pays concernés (16). Les tiques quant à elles provoquent des pertes directes (diminution de la croissance et de la production laitière, blessures pouvant se traduire par la destruction des trayons et ayant des conséquences importantes sur la vitalité des veaux avant sevrage) dues à leur action vulnérante et prédatrice (26). A. variegatum, l'espèce qui cause le plus de dégâts en Afrique de l'Ouest, est d'autre part liée à deux maladies qui affectent essentiellement les animaux exotiques introduits en zone d'enzootie, comme les bovins laitiers de races européennes. Il s'agit de la cowdriose (affection mortelle due à Ehrlichia ruminantium et dont la tique est le vecteur naturel) et de la dermatophilose, dont les lésions cutanées, souvent chroniques, sont aggravées par la présence des tiques adultes.

La connaissance du comportement des vecteurs, de leur biologie et de leur physiologie, permet le développement de méthodes de lutte spécifiques et adaptées. C'est ainsi que le fait que les glossines femelles ne s'accouplent qu'une fois puis stockent les spermatozoïdes qui les féconderont durant toute leur vie a permis la mise en place de la lutte par lâchers de mâles stériles $(8,9)$. Par ailleurs, la lutte stratégique contre la tique $A$. variegatum est possible en zone tropicale à saison des pluies unique, parce que les animaux ne sont fortement parasités par les tiques adultes que pendant les deux ou trois premiers mois de la saison pluvieuse $(17,22)$. D'autres observations, concernant les modalités d'attaque et d'envahissement des hôtes par les parasites, ont conduit à la mise au point par le Centre international de recherche-développement sur l'élevage en zone subhumide (Cirdes) d'une méthode de traitement peu onéreuse et rapide qui permet de lutter simultanément contre ces divers ectoparasites.

\section{- ETUDE DU COMPORTEMENT DES ECTOPARASITES}

\section{Amblyomma variegatum}

En zone tropicale, le cycle d'A. variegatum est totalement inféodé aux variations climatiques. Les adultes restent inactifs et cachés dans les microhabitats favorisant leur survie (anfractuosités du sol, lacis racinaire des arbustes et des touffes de graminées pérennes) tant que le taux d'humidité reste faible. Cette diapause comportementale est levée par les premières pluies. Les tiques sortent alors de terre et se mettent à l'affût dans les débris végétaux. Lorsque des bovins passent à proximité, les parasites sont activés et se dirigent vers les vertébrés sur lesquels ils cherchent à se fixer (20). Les zones de prédilection des adultes d'A. variegatum sont peu nombreuses : la plupart des tiques infestent le poitrail et la région inguinale. Le processus d'invasion de ces zones a été identifié grâce au marquage des tiques et au suivi pluriquotidien de l'infestation (24). Lorsqu'elles parviennent à rejoindre un bovin, les tiques se fixent immédiatement sur la peau des espaces interdigités, à proximité des onglons : environ 90 p. 100 des A. variegatum capturés chaque jour au pâturage par les animaux s'attachent ainsi sur ces sites de fixation provisoire. Les tiques restent fixées sur les pieds tant que les animaux restent debout. Comme, suivant les pratiques traditionnelles, les bovins, pâturant sept à neuf heures par jour en saison des pluies, ne peuvent que rarement se coucher au pâturage (quelques A. variegatum se fixent d'ailleurs sur les sites de prédilection sans passer par les pieds à la faveur de ces rares moments), la très grande majorité des tiques capturées dans la journée (87 à 93 p. 100 lors des divers essais réalisés) sont encore fixées sur les pieds des animaux le soir, lorsque ces derniers regagnent leur campement. C'est la nuit, quand les hôtes se reposent dans leur parc, que les tiques se détachent des sites de fixation provisoire pour rejoindre leurs sites de prédilection. Le traitement avec un acaricide de l'extrémité des membres élimine ainsi la plupart des tiques avant qu'elles ne se fixent sur ces sites, les dégâts causés étant alors encore très limités.

\section{Les glossines}

L'observation du comportement trophique de Glossina tachinoides et G. palpalis gambiensis (examen en étable sous moustiquaire des sites d'attaque et de repas de mouches marquées mises en présence d'un bovin entravé, les observateurs étant protégés par des moustiquaires) révèle que, chez les bovins, plus de 80 p. 100 des glossines s'alimentent au niveau des pattes, dont 40 p. 100 à leur extrémité (Bouyer et coll., résultats non publiés). Le tropisme d'attaque semble toutefois lié à l'espèce hôte et $G$. palpalis gambiensis piquerait en majorité les parties hautes du corps lorsqu'elle s'attaque à l'homme (19). Le tropisme pourrait également varier en fonction de la saison, la végétation masquant en partie l'extrémité des pattes pendant la saison des pluies (19). Cependant, l'impact du traitement pédiluve sur les glossines a été validé en saison humide sur le terrain (voir ci-dessous), confirmant le tropisme d'attaque observé en saison sèche sur les bovins.

En saison pluvieuse, les glossines se dispersent dans les savanes. C'est alors qu'il est intéressant d'utiliser le traitement épicutané du bétail qui constitue en l'occurrence un piège vivant pour ces insectes. Compte tenu du comportement trophique des tsé-tsé, la présence d'insecticide sur l'extrémité des pattes permet d'atteindre une grande partie de la population. De plus, les glossines ont un cycle de reproduction long et l'augmentation du taux de mortalité quotidien des femelles adultes au-dessus d'un seuil de 3,5 p. 100 suffit à assurer le déclin d'une population, jusqu'à son élimination si la pression de lutte est maintenue (13). En zone périurbaine, le biotope est fragmenté et les populations présentes dans ces mosaïques sont de petite taille, regroupant moins de 1000 individus : une lutte correctement conduite peut les affecter de façon très importante.

En saison sèche, en revanche, les glossines ripicoles se concentrent dans les zones au microclimat favorable, principalement les galeries forestières, et attaquent leurs hôtes, domestiques et sauvages, lorsqu'ils s'y aventurent, notamment pour boire. Il est donc possible de lutter à cette période de façon ciblée au niveau des points d'abreuvement par l'utilisation de pièges ou d'écrans imprégnés.

\section{Conséquences pour la lutte}

Le passage des bovins, au retour du pâturage, dans un pédiluve contenant une formulation acaricide/insecticide permet d'éliminer les Amblyomma variegatum adultes qui se sont fixés dans les espaces interdigités au cours de la journée. Du fait de la rémanence des produits utilisés, les tiques qui s'y attachent au cours de la ou des deux journées suivantes sont également tuées. D'autre part, le produit se propageant en partie sur les parties déclives du corps 
(région inguinale, poitrail, ventre...), à cause des éclaboussures inévitables lors du passage dans le pédiluve, des mouvements de la queue, et de la diffusion par contact lorsque les animaux sont couchés (les antérieurs touchent alors le poitrail), élimine également certaines des tiques déjà fixées sur les zones de prédilection.

Les glossines se posant sur les extrémités des membres, mais également une partie de celles qui piquent sur d'autres parties du corps (du fait de la diffusion du produit), sont paralysées et tombent au sol. Si la paralysie dure plus de six heures, les mouches sont détruites par les prédateurs. Or, les glossines sont en général beaucoup plus sensibles que les tiques aux produits acaricides/insecticides (rémanence de 30 jours contre 5 à 7 jours lors d'un traitement par une formulation de deltaméthrine à 0,005 p. 100 par exemple). Les traitements destinés à éliminer les tiques, effectués tous les deux ou trois jours, entraînent donc une mortalité élevée des glossines et par conséquent une prévention efficace des trypanosomoses animales.

\section{MISE EN OEUVRE DU TRAITEMENT}

PAR PEDILUVE

\section{Description du pédiluve}

Les pédiluves construits au Burkina Faso ont été décrits dans une fiche technique éditée par le Cirdes et disponible sur le site Internet du Centre (www.cirdes.org). Ils sont constitués d'un bac en béton armé mesurant $330 \mathrm{~cm}$ de long et $50 \mathrm{~cm}$ de large. La profondeur en est de $40 \mathrm{~cm}$. Les extrémités intérieures et extérieures du bac sont formées de plans inclinés, de façon à éviter que les bovins ne sautent et n'éclaboussent trop en y entrant ou en sortant, ce qui entrấnerait des pertes importantes de produit. Ce bac est installé dans un couloir précédé d'un parc d'attente permettant de canaliser les animaux vers le couloir. Il est enchâssé dans le sol mais le dépasse de $15 \mathrm{~cm}$ pour éviter l'entrée des eaux de ruissellement (figure 1).

Figure 1 : plans du pédiluve (mesures en centimètres).

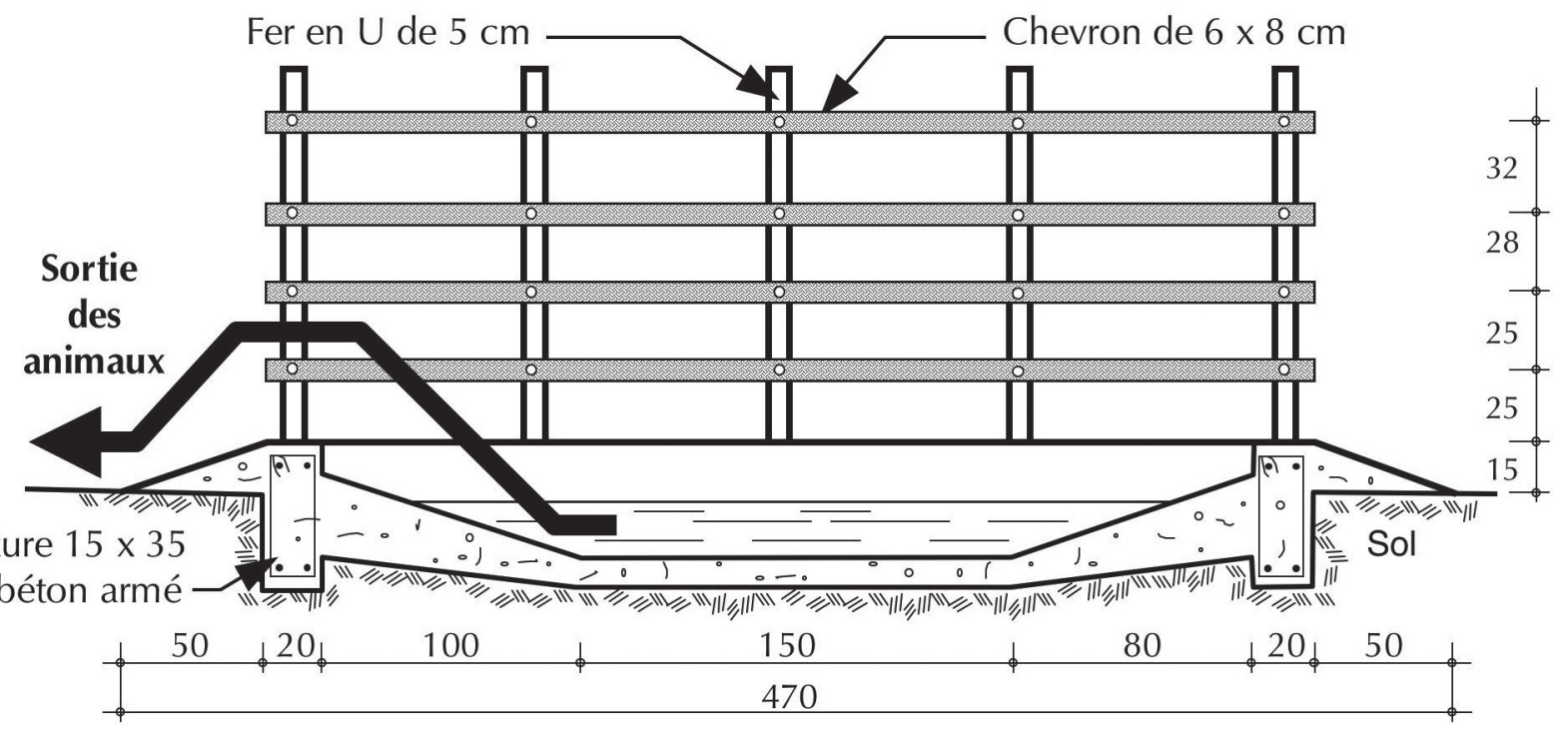

\section{Coupe longitudinale}

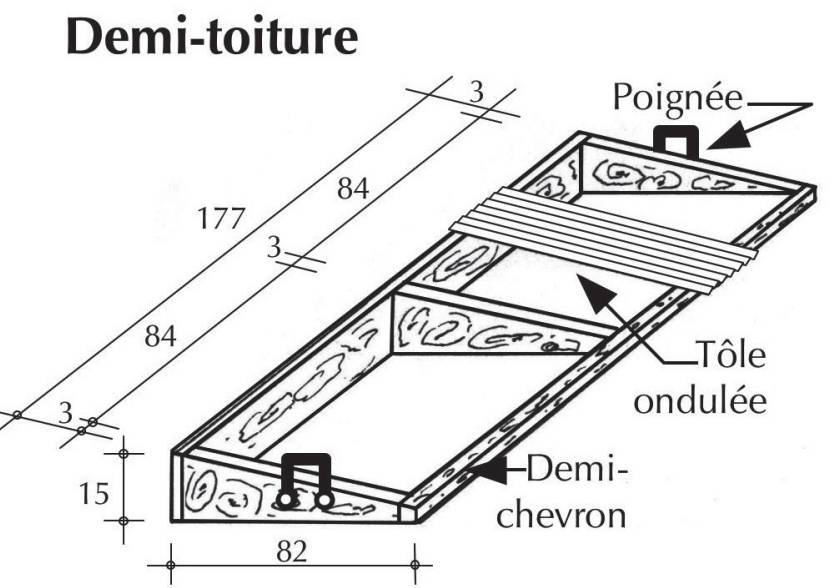

\section{Coupe transversale}

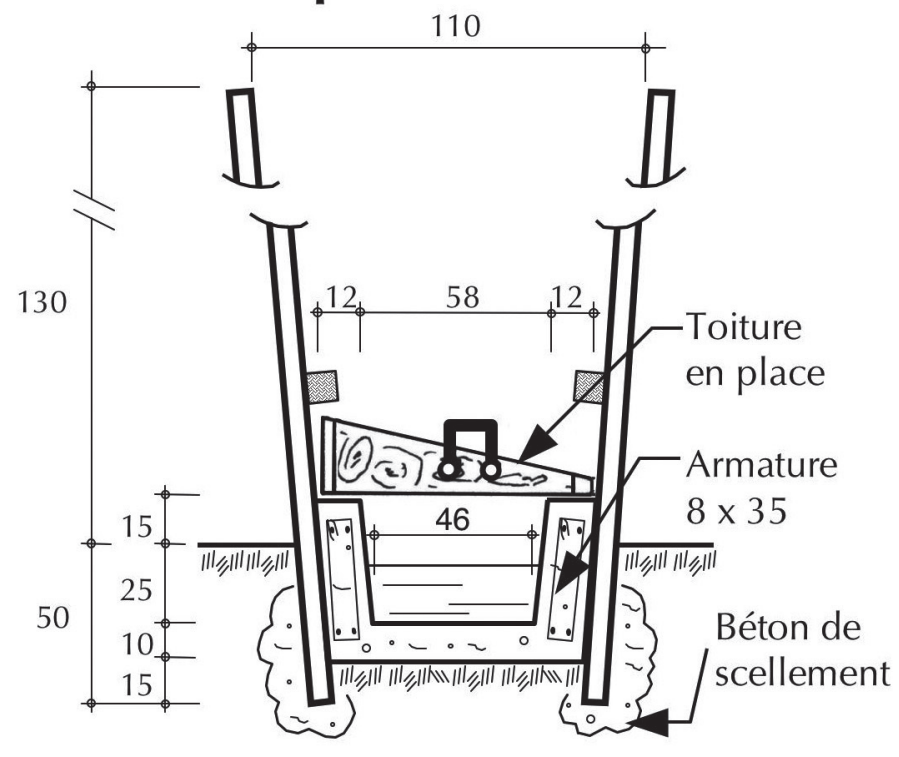


Lorsqu'il n'est pas utilisé, le bac est recouvert d'un toit (tôle ondulée fixée sur un cadre en bois) destiné à prévenir la dilution de la formulation aqueuse acaricide/insecticide par les pluies.

Rempli jusqu'à une hauteur de $20 \mathrm{~cm}$, le bac contient environ 2001 d'une formulation aqueuse de pyréthrinoïdes. Plusieurs principes actifs ont déjà été utilisés (fluméthrine, deltaméthrine, alphacyperméthrine), l'efficacité contre les tiques variant en fonction de la rémanence des produits (figure 2) qui est bien évidemment moindre que celle observée lorsque les acaricides sont appliqués sur le dos ou les flancs, car l'extrémité des membres est régulièrement trempée par la rosée, la traversée d'un marigot ou le contact avec la végétation mouillée par les pluies, ce qui tend à éliminer le principe actif.

\section{Utilisation du pédiluve}

Les recommandations suivantes doivent être suivies pour assurer le succès de ce mode de traitement : faire passer les animaux le soir, au retour du pâturage, avant qu'ils ne regagnent leur parc de nuit ; traiter les bovins pendant la période de forte infestation par les adultes d'A. variegatum, c'est-à-dire pendant les dix à douze premières semaines de la saison des pluies ; traiter tous les deux ou trois jours en fonction de la rémanence du produit utilisé et de l'infestation des pâturages ; ne commencer le traitement à ce rythme que lorsque chaque animal est infesté par environ une trentaine de tiques, ce qui évite les traitements non rentables ; maintenir par des ajouts réguliers d'eau et de produit (à la concentration recommandée par le fabricant) la hauteur de la formulation acaricide/insecticide dans le bac du pédiluve entre 15 et $20 \mathrm{~cm}$ pendant toute la période de traitement. Les ajouts pourront cesser une dizaine de jours avant la fin prévue du traitement, qui sera suspendu lorsque le niveau dans le bac sera trop faible (moins de $10 \mathrm{~cm}$ ). Après évaporation de l'eau, la boue résiduelle (quelques kilos) restant au fond du pédiluve sera laissée au soleil pendant plusieurs semaines, ce qui permettra l'inactivation du principe actif encore présent, avant d'être évacuée et dispersée aux alentours de l'installation.

Ne commencer le traitement que lorsque l'infestation moyenne des bovins atteint déjà quelques dizaines de tiques permet l'entretien

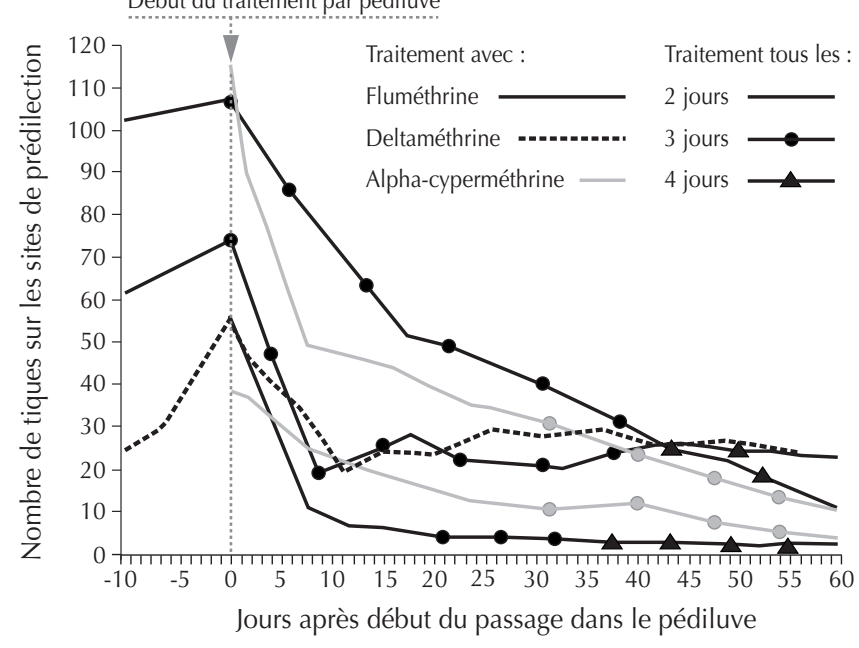

Figure 2 : évolution de l'infestation moyenne, par Amblyomma variegatum, des sites de prédilection de bovins, appartenant à six troupeaux, passant tous les deux à quatre jours dans un pédiluve contenant diverses formulations aqueuses de pyréthrinoïde. Dans certains cas, évolution de cette infestation avant la mise en place du traitement. de l'immunité protectrice vis-à-vis de la cowdriose (les races bovines locales bénéficient d'un statut de stabilité enzootique contre cette maladie) mais n'empêche en aucune façon la transmission de la maladie aux animaux sensibles, comme les bovins de races exotiques introduits en zone d'enzootie. Cette méthode de lutte vise ainsi spécifiquement à limiter les pertes directes dues aux tiques elles-mêmes, et non pas à prévenir la transmission d'Ehrlichia ruminantium, l'agent de la cowdriose. Il est en revanche probable que la dermatophilose, dont les lésions cutanées sont aggravées en présence des adultes d'A. variegatum du fait des propriétés immunosuppressives de la salive de la tique (28), sera au moins en partie endiguée par la forte diminution du nombre de tiques infestant les animaux. Cependant, le traitement par pédiluve n'ayant jusqu'alors été mis en œuvre que sur des animaux de races locales, peu sensibles à cette maladie, son efficacité dans la prévention de cette maladie ne peut être garantie.

En dehors de la période d'infestation par les A. variegatum adultes, traiter les animaux tous les cinq à sept jours permet de continuer à contrôler la population des glossines. Il est aussi possible d'utiliser, en saison sèche, une technique de lutte classique contre les mouches tsé-tsé (pièges ou écrans imprégnés ; 1, 5, 6) puisqu'elles se regroupent au niveau des galeries forestières.

\section{Résultats obtenus}

La lutte par pédiluve contre les adultes d'A. variegatum s'apparente à un traitement prophylactique dans la mesure où elle empêche la fixation définitive, sur les sites de prédilection, de la majorité des tiques capturées au pâturage. Pour être pleinement efficace, elle doit donc être réalisée très régulièrement. Les tiques fixées sur les pieds mais non éliminées se retrouvent en effet dès le lendemain sur les parties déclives du corps où elles sont moins facilement tuées par cette méthode.

L'examen régulier, tous les deux à cinq jours, d'animaux traités par pédiluve (comptage des tiques sur le corps de cinq à dix bovins choisis au hasard dans les troupeaux ; 25) a montré que leur infestation, après une diminution initiale, se maintenait à un niveau faible pendant toute la période de traitement, alors qu'elle augmentait régulièrement avant le passage des animaux dans le pédiluve (figure 2). Pourtant, dans la zone étudiée, les animaux capturaient en moyenne cinq à huit tiques par jour au pâturage (estimation faite par comptage des tiques présentes le soir sur les pieds), et l'infestation d'animaux non traités pâturant dans la même zone s'accroissait quotidiennement de quatre à six tiques. Lorsque le traitement est mis en place trop tardivement, alors que l'infestation initiale est déjà élevée (plus de 100 tiques par animal dans les cas présentés), la diminution de l'infestation est plus lente, témoignant de la capacité imparfaite de cette méthode à éliminer les parasites déjà en place (figure 2). Enfin, la présence permanente de produit sur l'extrémité des pattes est nécessaire au maintien d'un niveau d'infestation faible. Lorsque l'acaricide employé persiste moins longtemps (deltaméthrine sur la figure 2) ou lorsque les traitements sont trop espacés (passage tous les trois jours dans une formulation de fluméthrine pendant le pic d'infestation), l'infestation sera plus élevée que celle obtenue avec un traitement réalisé tous les deux jours avec un produit suffisamment rémanent (fluméthrine et alpha-cyperméthrine sur la figure 2).

Aux fréquences de passage usuelles contre les tiques (tous les deux ou trois jours), plus de 95 p. 100 des glossines sont paralysées par une formulation aqueuse d'alpha-cyperméthrine appliquée par cette méthode. Un tel résultat, dépassant les espérances liées aux sites d'attaque, est dû à la dispersion du produit sur le corps de l'animal lors des passages dans le pédiluve. Des essais en étable sous moustiquaire ont ainsi montré que le traitement par pédiluve 
avait une efficacité identique à celle d'un traitement par pulvérisation sur tout le corps de l'animal (figure 3). En dehors de la saison de forte infestation par les tiques, il est donc possible d'espacer les traitements, ou de combiner cette technique avec celles mises en œuvre de longue date (voir ci-dessus).

Si le traitement par pédiluve entraîne la paralysie de plus de 95 p. 100 des glossines trois jours après le dernier passage dans le pédiluve, il ne prévient que 35 p. 100 des attaques, une partie des glossines pouvant se gorger avant d'être paralysées. Cette protection partielle est valable pour tous les traitements épicutanés du bétail. Il y a donc un délai inévitable, nécessaire à la réduction de la population de glossines, avant que la diminution de la transmission des trypanosomes ne soit observée. Cette réduction n'est possible que dans les environnements à forte densité de bétail, où la majorité des animaux sont traités $(3,7,14,15,21)$. Si ces conditions sont respectées, on observe alors une réduction très rapide de l'incidence trypanosomienne (2). En zone périurbaine, les populations résiduelles de glossines sont en général peu abondantes et les contacts avec les animaux localisés à de rares points d'eau dont l'accès est encore autorisé par les agriculteurs. Dans une situation comme celle-ci, une augmentation du taux de mortalité quotidien de G. tachinoides et G. p. gambiensis de plus de 30 p. 100 a été observée, suite au traitement par pédiluve de 70 p. 100 des animaux utilisant un tel point d'eau, ce qui a conduit à une réduction significative de leurs densités (de 90 p. 100) après seulement quatre jours de traitement. L'incidence trypanosomienne mensuelle, qui a atteint 20 p. 100 en saison des pluies dans un troupeau témoin voisin du troupeau traité mais utilisant un autre point d'eau, a alors été significativement réduite, puis annulée après deux mois de traitement (Bouyer et coll., résultats non publiés).

Dans le cas des glossines riveraines en Afrique de l'Ouest, l'objectif de cette méthode est un contrôle prolongé. Une éradication est en effet improbable, y compris dans le cas d'une population de tsé-tsé isolée, en raison de la distribution hétérogène des mouches associée à un taux de mortalité variable en fonction de la localisation des sous-populations. Certaines d'entre elles se situent dans des zones refuges comme les bois sacrés où les bovins ne peuvent pénétrer : elles ne sont donc pas concernées par la lutte et constituent des poches réfractaires persistantes qui se nourrissent sur des hôtes sauvages tels que les varans. Seules sont éliminées les glossines situées dans des zones accessibles par le bétail : elles constituent les sous-populations «puits». Les sous-populations

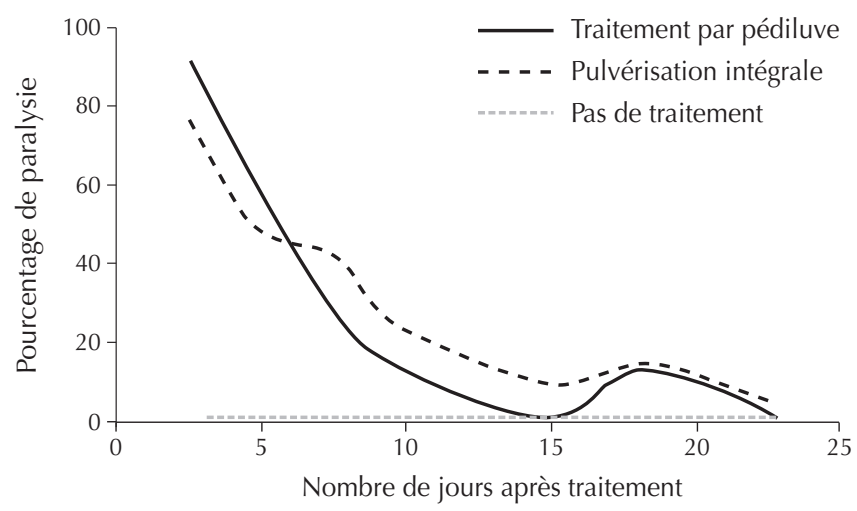

Figure 3 : taux de paralysie de Glossina palpalis gambiensis observé $2 h$ après recapture des mouches lâchées en présence d'un bovin en étable moustiquaire. Tous les trois à cinq jours, un lot de 100 mouches a été mis en présence d'un animal, traité ou non, puis suivi pendant plusieurs jours. qui vivent dans les poches réfractaires sont des sous-populations « sources », susceptibles de recoloniser rapidement toute la zone en cas d'arrêt du traitement. Ce phénomène est d'autant plus important qu'une glossine se nourrissant une première fois sur un varan aura tendance à retourner préférentiellement sur cette espèce par la suite (4). Dans le cas d'une population isolée, l'éradication pourrait cependant être obtenue par lâchers de mâles stériles, après une réduction des densités de plus de 99 p. 100 de la population initiale par l'utilisation du pédiluve $(9,23,27)$.

Le traitement par pédiluve est rapide (120 animaux peuvent être traités en moins d'un quart d'heure). Il est également peu onéreux : chaque animal emporte avec lui environ 200 à $250 \mathrm{ml}$ de mélange acaricide à chaque passage. Le traitement effectué régulièrement pendant toute la saison de forte infestation par les adultes d'A. variegatum revient alors à environ $150 / 200$ Fcfa $(0,23$ à $0,30 €)$. Par comparaison, un seul traitement par pour on revient à plus de $500 \mathrm{Fcfa}(0,75 €)$ et protége les animaux contre les tiques pendant une semaine à dix jours dans le meilleur des cas. Sur l'ensemble de la saison de forte infestation par la tique, le coût du traitement par pour on peut ainsi être estimé à environ 3000 à $4500 \mathrm{Fcfa}(4,6$ à $6,9 €)$. La pulvérisation hebdomadaire d'un animal revient, elle, à 75 Fcfa $(0,11 €)$, soit, pour toute la saison de forte infestation par la tique, 400 à $500 \mathrm{Fcfa}(0,6$ à $0,8 €)$, mais le traitement est bien plus long à mettre en œuvre, notamment pour les troupeaux de plusieurs dizaines d'individus. Comme le pédiluve est une installation fixe, il est particulièrement adapté aux troupeaux sédentaires ou semi-transhumants des agropasteurs. Les éleveurs transhumants pourraient cependant utiliser les installations présentes sur leur lieu de passage, à condition que les modalités en soient au préalable définies (tableau I).

Signalons enfin que d'autres ectoparasites attaquent les bovins essentiellement sur la partie distale des membres et seraient sans doute également touchés par la mise en place d'un traitement régulier par pédiluve. C'est le cas par exemple des stomoxes, mais aussi des moustiques, et notamment des anophèles vecteurs du paludisme. Il a en effet été observé que nombre d'entre eux piquaient aussi bien les humains que les bovins, préférant, chez ces derniers, les pattes (10). Le traitement ciblé des pieds des animaux pourrait ainsi avoir un impact non négligeable sur la transmission du plasmodium.

\section{INTERVENTION DES STRUCTURES D'APPUI}

\section{Justification}

Si le coût d'usage du pédiluve est faible, sa construction est en revanche assez onéreuse et revient à 350 à $400000 \mathrm{Fcfa}$ (530 à $610 €)$. Un pulvérisateur coûte pour sa part environ $60000 \mathrm{Fcfa}$ $(91 €)$ mais ne permet ni un traitement aussi rapide ni de traiter un nombre d'animaux aussi élevé. Le coût annuel de l'amortissement d'un pédiluve sera d'autant plus réduit qu'un plus grand nombre d'animaux l'utilisera, 400 à 600 animaux pouvant employer le même pédiluve (comme le traitement doit être réalisé le soir, au retour du pâturage, le pédiluve ne peut être utilisé que pendant 60 à 90 min chaque jour). C'est donc une installation qui sera construite avec profit par des groupements d'éleveurs lorsque les troupeaux sont regroupés géographiquement pendant la période de lutte (proximité des parcs de nuit). Les organisations professionnelles déclarées ont aussi l'avantage de pouvoir mobiliser des fonds et des aides plus facilement que les pasteurs traditionnels isolés.

Cet outil permet la mise en place d'une prestation de service gérée par les organisations professionnelles et concourt au renforcement technique et institutionnel de celles-ci. La mise en place et la pérennité du service reposent sur un bon fonctionnement institutionnel 


\section{Tableau I}

Canevas de cahier des charges liant une organisation professionnelle $(O P)$ d'éleveurs à une structure d'appui

\section{Rubrique}

Procédures de demande de mise en place de pédiluves

Modalités de l'appui financier

Modalités du choix

du lieu de construction

\section{Observations}

A détailler

A détailler

Proposition de l'OP d'un site selon les critères techniques et validation possible par un agent technique (préciser). Les critères techniques sont :

- sécurité foncière du terrain

- accessibilité du site pour les troupeaux identifiés (selon période)

- facilité d'accès et proximité des parcs de nuit

- absence de zone inondable pour le site et le trajet retour

- facilité d'apport d'eau pour l'entretien du pédiluve

Définition des utilisateurs

Par un organe institutionnel de l'OP et/ou avec contraintes.

Choix du gérant du pédiluve, fonctions, répartition des responsabilités et droits (formation, rétribution)

Gestion économique du service

Selon les lois de la vie associative/coopérative

Définition des contraintes (principes généraux de gestion, de composition du prix) et des éléments à déterminer selon le fonctionnement institutionnel de l'OP : prix exact du passage (membres/extérieurs), rétribution du gérant, fréquence de paiement...

Recommandations techniques,

Le protocole doit être défini (au moins partiellement) et les structures habilitées à contraintes techniques, assistance technique
I'adapter sont indiquées. Les responsabilités de l'OP intervenant dans l'encadrement technique sont définies. La structure d'appui forme (ou identifie) les structures ou personnes ressources pour assurer le suivi-conseil dans le long terme. de l'organisation : en effet, de nombreuses décisions et applications, comme le choix du site, le choix d'un gérant, la gestion économique du service, etc., seront déterminées dans le respect des principes de la vie associative/coopérative. La prophylaxie sanitaire devrait, d'autre part, davantage être envisagée comme une action collective (à mener à l'échelle du terroir en ce qui concerne la trypanosomose) vis-à-vis de laquelle un groupement a un rôle d'encadrement à jouer. Les éleveurs engagés dans une production de lait plus intensive, disposant de moyens plus importants et habitués à l'acquisition d'intrants et à la mise en œuvre de pratiques nouvelles, peuvent en revanche effectuer par eux-mêmes un tel investissement. Cependant, dans tous les cas de figure, l'encadrement par des techniciens s'avère nécessaire, au moins dans un premier temps, afin de familiariser les éleveurs, et plus particulièrement le gérant de l'installation, avec les contraintes de ce type de traitement et les conditions d'emploi : surveillance de la hauteur du mélange acaricide/insecticide, recharge du pédiluve en produit, comptage du nombre d'animaux fréquentant le pédiluve et de la fréquence de passage, calcul du coût $\mathrm{du}$ traitement, etc. Tous ces points doivent donner lieu à une formation spécifique assurée par les techniciens avant la mise en œuvre du traitement.

Certains éleveurs sont réticents à l'idée d'investir dans une technique nouvelle qui consiste à traiter uniquement les pieds pour éliminer des ectoparasites qui se fixent ou attaquent, pensent-ils, sur tout le corps. La construction d'installations pilotes permet de lever ces réticences par la démonstration du bien-fondé et de l'efficacité de la méthode. Là encore, les structures de développement peuvent intervenir afin de faciliter les transferts d'innovations.

\section{Actions possibles}

Les structures d'appui qui sont partenaires d'organisations professionnelles d'éleveurs, et qui sont convaincues de l'utilité de cette méthode de lutte contre les ectoparasites et favorables à sa mise en place, peuvent se baser, dans leur action, sur l'expérience menée au Burkina Faso et, dans un premier temps, sur certains documents édités par le Cirdes ${ }^{1}$.

Elles devraient ensuite envisager la réalisation d'ateliers de sensibilisation et de vulgarisation permettant de réunir les structures d'appui et les bénéficiaires autour d'une problématique technique, en l'occurrence la lutte contre les ectoparasites. Au cours de tels ateliers, une expertise scientifique est apportée aux agents de développement et alimente la réflexion entre les différents partenaires.

Une fois prise la décision de mettre en place des pédiluves, il faut donc envisager la construction d'installations pilotes. En milieu paysan, l'adoption réussie et avantageuse d'une nouvelle technique par les élevages voisins est très convaincante. L'impact des installations pilotes sera d'autant plus fort que le milieu est traditionnel. La visite d'installations pilotes rend l'outil et son fonctionnement plus concrets pour l'éleveur qui sera par exemple rassuré de voir

\footnotetext{
${ }^{1}$ Le pédiluve acaricide, fiche technique du Cirdes $\mathrm{n}^{\circ} 1,2004,8 \mathrm{p} . ;$ Traitement épicutané du bétail, fiche technique du Cirdes nº 8, 2004, 8 p. ; Actes de l'atelier de lutte contre la tique Amblyomma variegatum à l'aide de pédiluves acaricides, 24-25 mai 2004, Bobo-Dioulasso, Burkina Faso, 37 p. ; Lutte contre la tique Amblyomma variegatum, film vidéo sur cassette VHS présentant en détail la construction et l'utilisation du pédiluve (versions en français et en dioula), 2005, 33 minutes.
} 
le comportement des animaux l'utilisant. Il permet aussi d'approfondir les discussions sur des éléments techniques précis : la construction du pédiluve ou sa gestion par un groupement. L'installation pilote peut en effet, à court terme, être un pédiluve utilisé couramment par des éleveurs ne bénéficiant plus d'appui financier ou en nature (apport d'insecticide), éleveurs dont le témoignage serait la meilleure preuve de l'efficacité de la méthode.

\section{Points à maîtriser par les techniciens encadrant les éleveurs}

La formation de formateurs en production laitière et en élevage bovin (comme les techniciens d'élevage nationaux) à cet outil semble être un investissement particulièrement intéressant du fait de son impact durable. Le technicien assure une partie de la vulgarisation de la méthode et oriente les éleveurs encadrés. Son appui peut concerner aussi bien la gestion technique que la gestion économique du service. Ainsi il peut :

- valider le choix du site pour la construction ;

- calibrer un pédiluve nouvellement construit ;

- établir une grille donnant le volume différentiel entre deux mesures de hauteur de la solution;

- former le gérant à la lecture de la grille et au remplissage du tableau de passages des troupeaux;

- former le gérant à ajouter le volume de produit acaricide/insecticide nécessaire en fonction du niveau d'eau ajouté ;

- donner des conseils d'ordre général pour l'entretien du pédiluve ; - aider l'organisation professionnelle à calculer le prix du passage par unité animale; celui-ci se décompose en coût de revient de l'insecticide, en une marge pour la rétribution du gérant, et en une marge destinée à constituer un fonds de roulement pour les réparations éventuelles et l'amortissement du pédiluve ; une marge bénéficiaire aussi peut être envisagée ;

- être un premier interlocuteur face aux questions et difficultés des éleveurs.

Pour leur part, lorsqu'une aide financière intervient, les agents de développement ont pour charge de s'assurer de la bonne utilisation des fonds et du fonctionnement correct du service mis en place. L'élaboration d'un cahier des charges (tableau I), qui permet l'établissement d'un contrat moral et financier entre les différents protagonistes (les bénéficiaires de l'outil, la structure qui encadre les bénéficiaires, la structure d'appui technique et/ou financier...), s'avère un élément fondamental de la mise en œuvre de l'appui à des collectivités. Tous les aspects du fonctionnement financier et technique doivent être passés en revue dans le cahier des charges (sauf dans le cas d'un bénéficiaire unique comme un investisseur privé), afin d'établir les contraintes fixées par la structure d'appui, la répartition des responsabilités et les éléments devant être déterminés par les bénéficiaires eux-mêmes (par exemple le choix du gérant incombe au conseil de gestion s'il s'agit d'une coopérative ; tableau I).

\section{CONCLUSION}

La lutte contre les ectoparasites des bovins par pédiluve est une méthode innovante qui s'adresse aussi bien aux éleveurs intensifs de bovins en zone périurbaine qu'aux éleveurs traditionnels dont les troupeaux sont regroupés pendant la période de lutte contre les tiques. Elle possède de nombreux atouts comparativement aux méthodes déjà utilisées : si le contexte épidémiologique le permet, elle combine la lutte contre les tiques et contre les glossines ; sa mise en œuvre est rapide ; elle est peu onéreuse ; elle est facile à utiliser au quotidien et améliore les conditions de travail des éleveurs; elle permet la mise en place d'un nouveau service au sein des organisations professionnelles qui concourt au renforcement institutionnel de celles-ci ; elle renforce la dimension collective de la prophylaxie des maladies à vecteurs. Cependant, elle demande un investissement initial qui ne peut être apporté que par des investisseurs privés ou des organisations professionnelles d'éleveurs éventuellement appuyées par des bailleurs de fonds. Comme toute méthode innovante, un appui technique est souhaitable afin de favoriser son adoption. Cet appui peut passer par la diffusion des fiches techniques aux structures d'appui, par la réalisation d'ateliers de vulgarisation, par la construction d'installations pilotes, par la formation de formateurs et par le suivi technique des structures de développement.

\section{BIBLIOGRAPHIE}

1. BAUER B., AMSLER-DELAFOSSE S., KABORE I., KAMUANGA M. 1999. Improvement of cattle productivity through rapid alleviation of African trypanosomosis by integrated disease management practices in the agropastoral zone of Yalé, Burkina Faso. Trop. Anim. Health Prod., 31: 89-102.

2. BAUER B., KABORE I., LIEBISCH A., MEYER F., PETRICH-BAUER J. 1992. Simultaneous control of ticks and tsetse flies in Satiri, Burkina Faso, by the use of flumethrin pour on for cattle. Trop. Med. Parasitol., 43: 41-46.

3. BAYLIS M., STEVENSON P., 1998. Trypanosomiasis and tsetse control with insecticidal pour-ons: fact and fiction? Parasitol. Today, 14: 77-82.

4. BOUYER J., CUISANCE D., MESSAD S., GUERIN P.M., 2005. Learning affects host preference in tse-tse flies. Revue Élev. Méd. vét. Pays trop., 58: $27-29$.

5. CUISANCE D., 1989. Le piégeage des tsé-tsé. Maisons-Alfort, France, Cirad-lemvt, 172 p. (Etudes et synthèses $n^{\circ} 32$ )

6. CUISANCE D., BARRE N., DE DEKEN R., 1994. Ectoparasites des animaux : méthodes de lutte écologique, biologique, génétique et mécanique. Revue sci. tech. Off. int. Epizoot., 13 : 1305-1356.

7. CUISANCE D., BOUTRAIS J., 1995. Evaluation de la situation et de la stratégie de lutte contre les glossines et les trypanosomoses dans I'Adamaoua (Cameroun). Montpellier, France, Cirad-emvt, 63 p.

8. CUISANCE D., POLITZAR H., CLAIR M., SELLIN E., TAZE Y., 1978. Impact des lâchers de mâles stériles sur les niveaux de deux populations sauvages de Glossina palpalis gambiensis en Haute-Volta (sources de la Volta Noire). Revue Elev. Méd. vét. Pays trop., 31 : 315-328.

9. CUISANCE D., POLITZAR H., MEROT P., TAMBOURA I., 1984. Les lâchers de mâles irradiés dans la campagne de lutte intégrée contre les glossines dans la zone pastorale de Sidéradougou, Burkina Faso. Revue Elev. Méd. vét. Pays trop., 37 : 449-467.

10. HABTEWOLD T., PRIOR A., TORR S.J., GIBSON G., 2004. Could insecticide-treated cattle reduce Afrotropical malaria transmission? Effects of deltamethrin-treated zebu on Anopheles arabiensis behaviour and survival in Ethiopia. Med. vet. Entomol., 18: 408-417.

11. HAMADOU S., KAMUANGA M., ABDOULAYE A.T., LOWENBERG DEBOER J., 2005. Facteurs affectant I'adoption des cultures fourragères dans les élevages laitiers périurbains de Bobo-Dioulasso (Burkina Faso). Tropicultura, 23 : 29-35.

12. HAMADOU S., MARICHATOU H., KAMUANGA M., 2003 Croissance désordonnée des élevages périurbains et approvisionnemen de la ville de Bobo-Dioulasso : problématique de I'hygiène du lait. Etud. rech. sahéliennes, 8-9: 107-115.

13. HARGROVE J.W., 2003. Tsetse eradication: sufficiency, necessity and desirability. Edinburgh, UK, Centre for Tropical Veterinary Medicine, $134 \mathrm{p}$.

14. HARGROVE J.W., OMOLO S., MSALILWA J.S.I., FOX B., 2000. Insecticide-treated cattle for tsetse control: the power and the problems. Med. Vet. Entomol., 14: 123-130.

15. HARGROVE J.W., TORR S.J., KINDNESS H.M., 2003. Insecticidetreated cattle against tsetse (Diptera: Glossinidae): what governs success? Bull. entomol. Res., 93: 203-217.

16. ITARD J., CUISANCE D., TACHER G., 2003. Trypanosomoses historique - répartition géographique. Principales maladies infectieuses et parasitaires du bétail. Europe et régions chaudes. Paris, France, Lavoisier, p. 1607-1615.

17. KAISER M.N., SUTHERST R.W., BOURNE A.S., GORISSEN L., FLOYD R.B., 1988. Population dynamics of ticks on Ankole cattle in five ecological zones in Burundi and strategies for their control. Prev. vet. Med., 6: 199-222. 
18. MARICHATOU H., HAMADOU S., KANWE B.A., 2003. Production laitière dans les systèmes d'élevage peri-urbains en zone subhumide du Burkina : situation et voies d'amélioration. Etud. rech. sahéliennes, 8-9 : 89-97

19. NASH T.A.M., 1948. Tsetse flies in British West Africa. London, UK, His Majesty's stationery office, $260 \mathrm{p}$.

20. NORVAL R.A.I., YUNKER C.E., BUTLER J.F., 1987. Field sampling of unfed adults of Amblyomma hebraeum Koch. Exp. Appl. Acarol., 3: 213-217.

21. OKIRIA R., OKUNA N.M., MAGONA J.W., MAYENDE J.S.P., 2002 Sustainability of tsetse control by subsequent treatment of $10 \%$ of a previously treated Ugandan cattle population with $1 \% \mathrm{w} / \mathrm{v}$ deltamethrin. Trop. Anim. Health Prod., 34: 105-114.

22. PETNEY T.N., HORAK I.G., RECHAV Y., 1987. The ecology of the African vectors of heartwater, with particular reference to Amblyomma hebraeum and A. variegatum. Onderstepoort J. vet. Res., 54: 381-395.

23. POLITZAR H., CUISANCE D., 1984. An integrated campaign agains riverine tsetse flies Glossina palpalis gambiensis and Glossina tachinoides by trapping and the release of sterile males. Insect Sci. Appl., 5: 439-442.
24. STACHURSKI F., 2000. Invasion of West African cattle by the tick Amblyomma variegatum. Med. vet. Entomol., 14: 391-399.

25. STACHURSKI F., LANCELOT R., 2006. Footbath acaricide treatment to control cattle infestation by the tick Amblyomma variegatum. Med. vet. Entomol. (in press)

26. UILENBERG G., 1992. Veterinary significance of ticks and tick-borne diseases. In: Fifaz B., Petney T., Horzk I. Eds, Tick vector biology. Medical and veterinary aspects. Berlin, Germany, Springer-Verlag, p. 23-33.

27. VREYSSEN M.J.B., SALEH K.M., ALI M.Y., ABDULLA A.M., ZHU Z.R., JUMA K.G., DYCK V.A., MSANGI A.R., MKONVI P.A., FELDMANN H.U., 2000. Glossina austeni (Diptera: Glossinidae) eradicated on the Island of Unguja, Zanzibar, using the sterile insect technique. J. Econ. Entomol., 93: 123-135.

28. WALKER A.R., LLOYD C.M., 1993. Experiments on the relationship between feeding of the tick Amblyomma variegatum (Acari: Ixodidae) and dermatophilosis skin disease in sheep. J. Med. Entomol., 30: 136-143.

Reçu le 06.03.2006, accepté le 14.06.2006

\section{Summary}

Stachurski F., Bouyer J., Bouyer F. Innovative Method to Control Cattle Ectoparasites in Suburban Areas of the Subhumid Zone of Burkina Faso: the Footbath

A consequence of the increase of the urban demand for animal proteins is the development of the dairy cattle subsector in suburban areas of West Africa. But the inadequate control of diseases and parasites hampers production increase. A novel control method, based on cattle passing through a footbath containing an aqueous formulation of a pyrethroid, could however limit the impact of ticks and tsetse flies, the main pathologic constraints in subhumid areas. This method leads to the elimination of most of adult Amblyomma variegatum, the most harmful tick species in these areas, before attachment to their predilection sites. Furthermore, the footbath treatment helps to reduce drastically the most common populations of riverine tsetse flies in West Africa (Glossina tachinoides and G. palpalis gambiensis), and therefore helps limit trypanosomosis incidence. This control method is also efficacious, low timeconsuming and relatively unexpensive, because the product quantity used in the footbath at each passage is low. But the construction of the structure, which can be used by 400 to 600 head of cattle, is rather expensive and can be more easily achieved by farmers' associations or private investors than by traditional farmers on their own. This method could be implemented within the framework of planning and development policies. The modalities of the technical support needed are described. In Burkina Faso, a development project for dairy cattle production (ARIOPE) financed the construction of 15 footbaths in the suburban areas of Bobo-Dioulasso and Ouagadougou.

Keywords: Cattle - Metastigmata - Glossinidae - Control method - Integrated control - Group approach - Innovation Burkina Faso.

\section{Resumen}

Stachurski F., Bouyer J., Bouyer F. Lucha contra los ectoparásitos de los bovinos mediante pediluvios: método innovador utilizado en la zona peri urbana sub húmeda de Burkina Faso

El aumento en la demanda urbana en proteínas animales se traduce, entre otras cosas, por el desarrollo de filiales bovinas de producción lechera en las zonas peri urbanas de Africa del Oeste. Pero el control imperfecto de la patología compromete el crecimiento de la producción. Una técnica de lucha innovadora, basada sobre el paso regular en un pediluvio que contiene una fórmula acuosa de piretroide, podría por en tanto limitar el impacto de las garrapatas y de las glosinas, principales obstáculos patológicos en la zona sub húmeda. Este método ocasiona la eliminación de la mayoría de los adultos de Amblyomma variegatum, la garrapata más nociva en estas regiones, antes de su fijación definitiva. Por otra parte, este tratamiento permite disminuir fuertemente la población de las glosinas ribereñas más representadas en Africa occidental (Glossina tachinoides et G. palpalis gambiensis) y limitando así la incidencia de tripanosomoses. Este método permite así una lucha rápida y eficaz a costo reducido, debido a que la cantidad de producto utilizado en cada pasaje es baja. Pero la construcción de las instalaciones, que pueden ser utilizadas por 400 a 600 animales, representa una inversión importante, que los grupos de criadores o de inversionistas privados pueden considerar más fácilmente que los criadores tradicionales aislados. La realización de este método puede ser entonces considerada dentro del marco de una política de desarrollo y mejoramiento. El interés y las modalidades del apoyo técnico necesario están presentes. En Burkina Faso, un proyecto de apoyo al refuerzo de las organizaciones profesionales de criadores modernos (Ariope) financió de esta manera 15 instalaciones en las zonas peri urbanas de Bobo-Diulasso y Uagadudu.

Palabras clave: Ganado bovino - Metastigmata - Glossinidae Método de control - Lucha integrada - Enfoque de grupo Innovación - Burkina Faso. 